\title{
Hepatoprotective effects of berberine on carbon tetrachloride-induced acute hepatotoxicity in rats
}

\author{
Yibin Feng ${ }^{1 *}$, Ka-Yu Siu' ${ }^{1}$ Xingshen Ye ${ }^{1}$, Ning Wang ${ }^{1}$, Man-Fung Yuen ${ }^{2}$, Chung-Hang Leung ${ }^{3}$, Yao Tong ${ }^{1}$, \\ Seiichi Kobayashi ${ }^{4}$
}

\begin{abstract}
Background: Berberine is an active compound in Coptidis Rhizoma (Huanglian) with multiple pharmacological activities including antimicrobial, antiviral, anti-inflammatory, cholesterol-lowering and anticancer effects. The present study aims to determine the hepatoprotective effects of berberine on serum and tissue superoxide dismutase (SOD) levels, the histology in tetrachloride $\left(\mathrm{CCl}_{4}\right)$-induced liver injury.

Methods: Sprague-Dawley rats aged seven weeks were injected intraperitoneally with $50 \% \mathrm{CCl}_{4}$ in olive oil. Berberine was orally administered before or after $\mathrm{CCl}_{4}$ treatment in various groups. Twenty-four hours after $\mathrm{CCl}_{4}$ injection, serum alanine aminotransferase (ALT) and aspartate aminotransferase (AST) activities, serum and liver superoxide dismutase (SOD) activities were measured. Histological changes of liver were examined with microscopy.
\end{abstract}

Results: Serum ALT and AST activities significantly decreased in a dose-dependent manner in both pre-treatment and post-treatment groups with berberine. Berberine increased the SOD activity in liver. Histological examination showed lowered liver damage in berberine-treated groups.

Conclusion: The present study demonstrates that berberine possesses hepatoprotective effects against $\mathrm{CCl}_{4^{-}}$ induced hepatotoxicity and that the effects are both preventive and curative. Berberine should have potential for developing a new drug to treat liver toxicity.

\section{Background}

Liver damage induced by carbon tetrachloride $\left(\mathrm{CCl}_{4}\right)$ involves biotransformation of free radical derivatives, increased lipid peroxidation and excessive cell death in liver tissue [1,2]. This model of $\mathrm{CCl}_{4}$-induced liver injury has been widely used in new drug development for liver diseases.

Berberine is a plant alkaloid present in many medicinal herbs, such as Hydrastis canadensis, Coptidis Rhizoma, Berberis aquifolium, Berberis aristata and Berberis vulgaris [3]. Coptidis Rhizoma (Huanglian), which is rich in berberine, exhibited hepatoprotective effects on $\mathrm{CCl}_{4}$-induced liver injury via scavenging the peroxidative products [4]. Antioxidative effects of Coptidis Rhizoma and its major active ingredient berberine against peroxynitrite-induced kidney damage were

\footnotetext{
* Correspondence: yfeng@hku.hk

'School of Chinese Medicine, The University of Hong Kong, 10 Sassoon

Road, Pokfulam, Hong Kong SAR, China

Full list of author information is available at the end of the article
}

demonstrated in vitro and in vivo [5]. Previous studies reported that berberine inhibited inflammation [6] and low-density lipoprotein (LDL) oxidation [7]. Other studies found that berberine was a candidate drug for Alzheimer's disease [8] and cancer [9]. Berberine exhibited no curative action on $\mathrm{CCl}_{4}$-induced liver injury whereas serum alanine aminotransferase (ALT) and aspartate aminotransferase (AST) levels were ameliorated after berberine treatment [10]. It is interesting that we showed in our previously study Coptidis Rhizoma exhibits curative effect of $\mathrm{CCl}_{4}$-induced liver injury in rats, which is discrepant to the reference reports since berberine is considered as the major active compound in Coptidis Rhizoma [4]. To clarify the gap and discrepancy among the above reports, it is necessary to do a more systematic and comprehensive study on hepatoprotective effects of bererbine in CCl4-induced acute liver toxicity.

The present study aims to examine the preventive and curative effects of berberine on liver injury and serum,
C Biomed Central

(c) 2010 Feng et al; licensee BioMed Central Ltd. This is an Open Access article distributed under the terms of the Creative Commons Attribution License (http://creativecommons.org/licenses/by/2.0), which permits unrestricted use, distribution, and reproduction in any medium, provided the original work is properly cited. 
tissue superoxide dismutase (SOD) levels and the tissue histology.

\section{Methods}

Drugs and chemical reagents

Berberine, $\mathrm{CCl}_{4}$ Heparin, Phenobarbital and olive oil were obtained from Sigma (USA). ALT and AST test kits were purchased from Stanbio (USA). SOD assay kit was obtained from Dojindo Laboratories (Japan).

\section{Animals}

Male Sprague-Dawley rats aged 7 weeks weighing 230$270 \mathrm{~g}$ were obtained from the Laboratory Animal Centre of the University of Hong Kong. Animals were allowed to acclimate for two days; they were fed with standard pellet diet and water ad libitum at $20-25^{\circ} \mathrm{C}$ under a 12 hour light/dark cycle. Food was withdrawn one day before the experiment but water continued to be provided.

All animal handlings and experiment protocols complied with the guidelines of the Laboratory Animal Centre of the University of Hong Kong. Animals were processed (including drug treatment and sacrifice) in accordance with the international guidelines for laboratory animals.

\section{$\mathrm{CCl}_{4}$-induced acute liver damage model}

48 animals were divided into six groups, namely Group 1: control group, Group 2: $\mathrm{CCl}_{4}$ control group, Group 3: low dose treatment group (post-treated with berberine, $80 \mathrm{mg} / \mathrm{kg}$ ), Group 4: medium dose treatment group (post-treated with berberine, $120 \mathrm{mg} / \mathrm{kg}$ ), Group 5: high dose treatment group (post-treated with berberine, $160 \mathrm{mg} / \mathrm{kg}$ ) and Group 6: preventive dose treatment group (pre-treated with berberine, $120 \mathrm{mg} / \mathrm{kg}$ ). Each group contained eight animals. Rats from Groups 2 to 6 were intraperitoneally (ip) injected with $\mathrm{CCl}_{4}$ at a dose of $1.0 \mathrm{ml} / \mathrm{kg}$ as a $50 \%$ olive oil solution while Group 1 received $1.0 \mathrm{ml} / \mathrm{kg}$ of olive oil. Berberine was suspended in distilled water at concentrations of 80,120 and $160 \mathrm{mg} / \mathrm{kg}$ which were orally administered through a stomach tube to rats in Groups 3 to 5 respectively after six hours of $\mathrm{CCl}_{4}$ treatment. Rats in Group 6 were orally administered with berberine $(120 \mathrm{mg} / \mathrm{kg})$ twice daily for two days before $\mathrm{CCl}_{4}$ treatment. The $\mathrm{CCl}_{4}$ control group (Group 2) was orally administered with distilled water of the equivalent volume.

Twenty-four (24) hours after $\mathrm{CCl}_{4}$ administration, the animals were anesthetized with ketamine/xylazine mixture (ketamine $67 \mathrm{mg} / \mathrm{kg}$, xylazine $6 \mathrm{mg} / \mathrm{kg}$, ip). Blood samples were collected by cardiac puncture, placed in heparinized tubes and centrifuged at $3000 \times \mathrm{g}$ (Eppendorf, Germany) for 10 minutes to obtain sera which were used to determine SOD and to test ALT and AST activities.
Immediately after blood collection, the animals were sacrificed by an overdose of pentobarbitone (Phenobarbital $200 \mathrm{mg} / \mathrm{kg}$, ip). The liver of each rat was promptly removed and used to determine the tissue level SOD and for further histopathological study.

\section{Serum ALT and AST analyses}

ALT and AST activities in serum samples were measured with Stanbio kits and a UV-rate auto-analyzer (Hitachi 736-60, Japan).

Values of the serum ALT and AST activities were derived according to the 'absorptivity micromolar extinction coefficient' of NADH at $340 \mathrm{~nm}$ and were expressed in terms of unit per liter (U/L). One unit per liter was defined as the amount of enzyme required to oxidize one $\mu \mathrm{mol} / \mathrm{L}$ of NADH per minute.

\section{Measurement of serum SOD}

Serum SOD was determined according to the technical manual of the SOD assay kit-WST (Dojindo Laboratories, Japan).

Briefly, the assay kit utilized the mitochondrial activity producing a water-soluble formazan dye upon reduction with the superoxide anion. The rate of the reduction with a superoxide anion was linearly related to the xanthine oxidase (XO) activity and was inhibited by SOD. Thus, the inhibition rate of XO activity determined by a colorimetric method was used to reflect the serum SOD levels in this study.

\section{Histopathological analysis}

Liver samples were immediately collected and fixed in $10 \%$ buffered formaldehyde solution for a period of at least 24 hours before histopathological study. Samples were then embedded in paraffin wax with Automatic Tissue Processor (Lipshaw, USA) and five-micron sections were prepared with a Leica RM 2016 rotary microtome (Leica Instruments, China). These thin sections were stained with hematoxylin and eosin (H\&E) and mounted on glass slides with Canada balsam (Sigma, USA). Degrees of liver damage were estimated as described before[4] under a light microscope (Leica Microsystems Digital Imaging, Germany) and images were captured with a Leica DFC 280 CCD camera (Leica, Germany) at original magnification of $10 \times 10$. The grades of liver damage in different groups were assigned in numerical scores (scale from 0 to 6).

\section{Statistical analysis}

Data were presented as mean and standard deviation (SD). When one-way ANOVA showed significant differences among groups, Tukey's post hoc test was used to determine the specific pairs of groups that were statistically different. A level of $P<0.05$ was considered 
statistically significant. Analysis was performed with the software SPSS version 16.0 (SPSS Inc, USA).

\section{Results}

Effects of berberine post-treatment on serum ALT and AST activities

Effects of berberine on serum ALT and AST activities in rats from various treatment groups are shown in Figure 1. After 24 hours of $\mathrm{CCl}_{4}$ treatment, the serum ALT and AST activities increased significantly (ALT: $F=11.5$, $\mathrm{P}<0.001$; AST: $\mathrm{F}=12.8, P<0.001)$. Serum ALT and AST activities in berberine co-treatment groups of 'Low dose', 'Medium dose' and 'High dose' decreased significantly in a dose-dependent manner (ALT: Low: $\mathrm{F}=7.3, P<0.001$; Medium: $\mathrm{F}=10.3, P<0.001$; High: $\mathrm{F}=11.3, P<0.001$; AST: Low: $\mathrm{F}=7.4, P<0.001$; Medium: $\mathrm{F}=12.8$, $P<0.001$; High: $\mathrm{F}=13.8, P<0.001$ when compare when $\mathrm{CCl}_{4}$ group). Both medium and high doses of berberine suppressed the ALT and AST activities up to or lower than the level in normal rats (ALT: Medium: $\mathrm{F}=1.2$; $P=0.254$; High: $\mathrm{F}=0.1, \mathrm{P}=0.906$; AST: Medium: $\mathrm{F}=0.0$, $P=0.999$; High: $\mathrm{F}=1.0, P=0.316$ when compared with normal group)

\section{Effects of berberine post-treatment on serum SOD activity}

Effects of berberine on serum SOD activity in various treatment groups are shown in Figure 2. After 24 hours of $\mathrm{CCl}_{4}$ treatment, serum SOD activity decreased significantly $(\mathrm{F}=23.8, P<0.001)$ and the serum SOD level in berberine co-treatment groups of 'Low' and 'Medium' and 'High' increased significantly in a dose-dependent manner (Low: $\mathrm{F}=4.5, P<0.001$; Medium: $\mathrm{F}=13.5$,

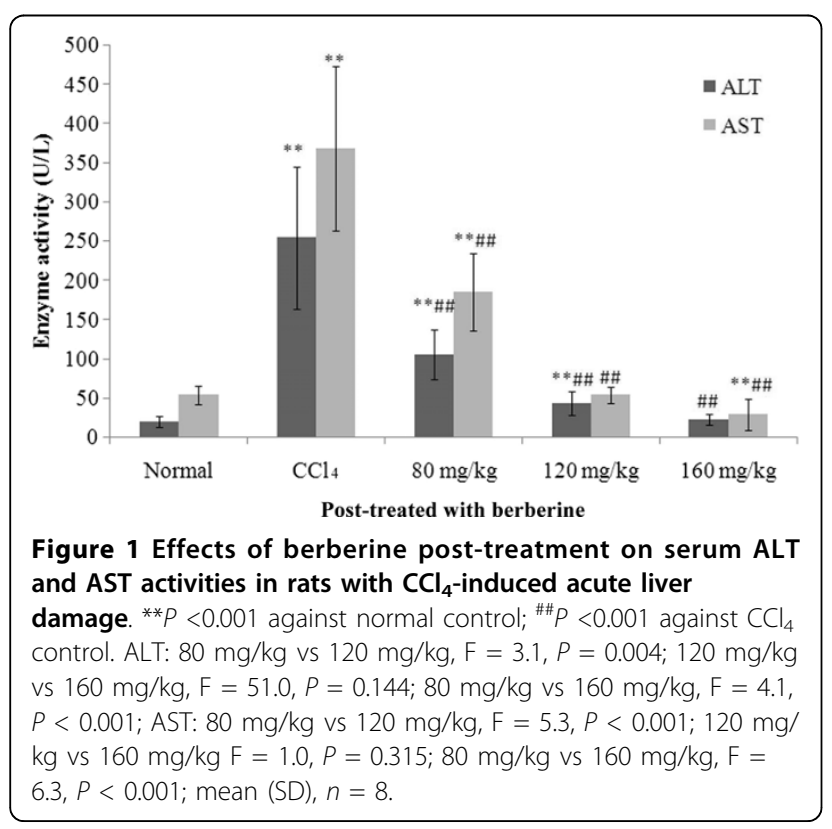

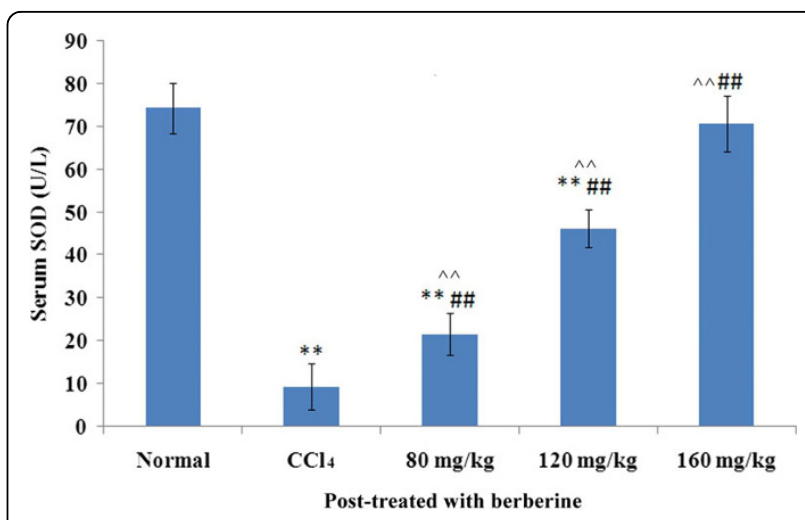

Figure 2 Effects of berberine post-treatment on serum SOD activity in rats with $\mathrm{CCl}_{4}$-induced acute liver damage. ${ }^{*} P$

$<0.001$ against normal control; ${ }^{\# \#} P<0.001$ against $\mathrm{CCl}_{4}$ control and $\wedge \wedge P<0.001$ among three different dosages; mean (SD), $n=8$. SOD: $80 \mathrm{mg} / \mathrm{kg}$ vs $120 \mathrm{mg} / \mathrm{kg}, \mathrm{F}=9.0, P<0.001 ; 120 \mathrm{mg} / \mathrm{kg}$ vs $160 \mathrm{mg} /$ $\mathrm{kg}, \mathrm{F}=8.9, P<0.001 ; 80 \mathrm{mg} / \mathrm{kg}$ vs $160 \mathrm{mg} / \mathrm{kg}, \mathrm{F}=18.0, P<0.001 ;$ mean (SD), $n=8$.

$\mathrm{P}<0.001$; High: $\mathrm{F}=22.5, P<0.001$ when compared with $\mathrm{CCl}_{4}$ group). The high dose group $(160 \mathrm{mg} / \mathrm{kg}$ berberine) showed normal SOD level ( $\mathrm{F}=1.4, P=0.173$ when compared with normal group) which was the best among the three berberine treatment groups.

\section{Effects of berberine pre-treatment on serum ALT and AST} activities

Effects of berberine pre-treatment on serum ALT and AST activities in rats treated with $\mathrm{CCl}_{4}$ at a dose of 1.0 $\mathrm{ml} / \mathrm{kg}$ are shown in Figure 3. Serum ALT and AST activities in rats pre-treated with berberine were significantly lower than those in rats treated with $\mathrm{CCl}_{4}$ (ALT: $\mathrm{F}=8.8, P<0.001 ;$ AST: $\mathrm{F}=12.0, P<0.001)$.

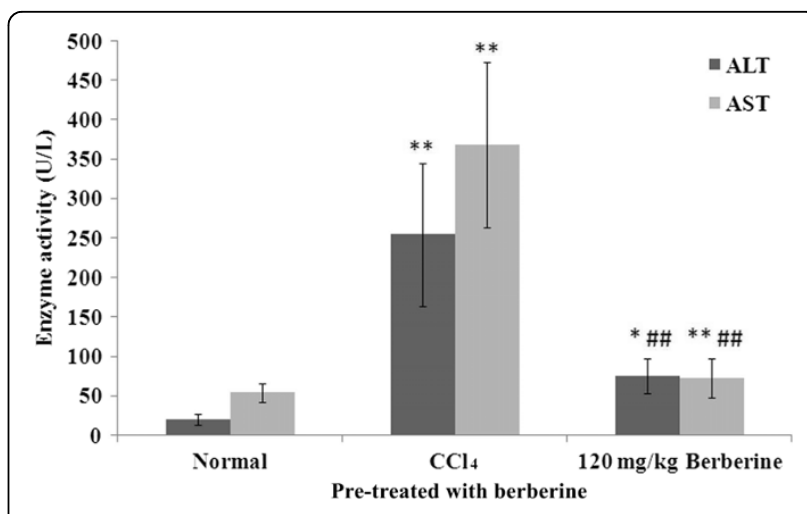

Figure 3 Effects of berberine pre-treatment on serum ALT and AST activities in rats with $\mathrm{CCl}_{4}$-induced acute liver damage. ${ }^{*} P$ $<0.01$ vs normal control; ${ }^{* *} P<0.001$ vs normal control; ${ }^{\#} P<0.001$ vs $\mathrm{CCl}_{4}$ control; mean (SD), $n=8$. 
Effects of berberine pre-treatment on serum SOD activity Effects of berberine pre-treatment on serum SOD activity of rats are shown in Figure 4. While the serum SOD activity in rats from berberine pre-treatment was significantly lower than that in normal rats ( $\mathrm{F}=12.9, P<$ $0.001)$, it was much higher than that in rats treated with $\mathrm{CCl}_{4}(\mathrm{~F}=10.9, P<0.001)$.

\section{Histology}

Results from the histological studies were in agreement with the measured activities of serum enzymes. There were no abnormalities or histological changes in the livers of normal rats (Figure 5a). Severe hepatocyte necrosis, inflammatory cells infiltration, fatty degeneration, hemorrhage and hydropic degeneration were found in rats 24 hours after $\mathrm{CCl}_{4}$ treatment (Figure 5b). Vacuole generation and microvascular steatosis were also observed. Post-treatment of berberine at 160, 120 and $80 \mathrm{mg} / \mathrm{kg}$ reduced the severity of $\mathrm{CCl}_{4}$-induced liver intoxication (Figures 5c, d and 5e). Fatty change, necrosis and lymphocyte infiltration were improved in the histological sections of berberine post-treated rats. Pretreatment of berberine before $\mathrm{CCl}_{4}$ intoxication also attenuated the hepatic damage induced by $\mathrm{CCl}_{4}$ (Figure $5 \mathrm{f})$. These results indicated the effects of berberine against $\mathrm{CCl}_{4}$-induced acute liver damage in a dosedependent manner (Table 1).

\section{Discussion}

In the present study the $\mathrm{CCl}_{4}$ treatment alone and posttreatment after 24 hours caused severe acute liver damage in rats, as evidenced by increased serum ALT and AST activities and a decreased serum SOD level

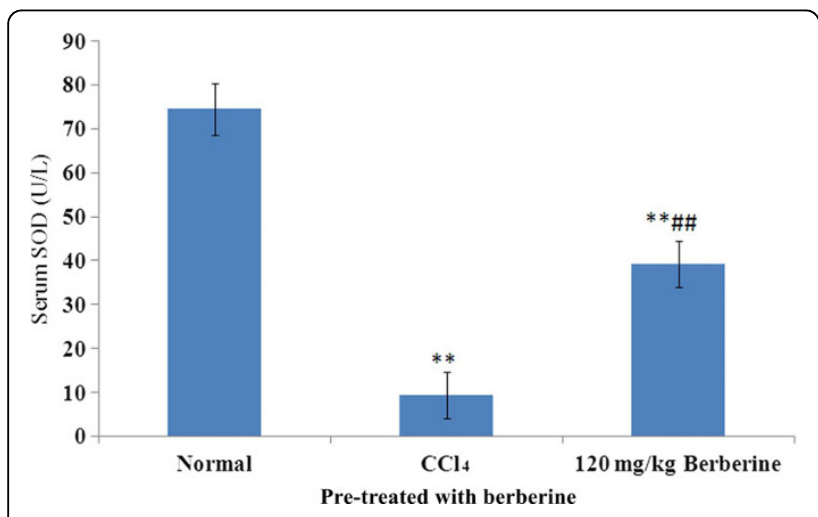

Figure 4 Effects of berberine pre-treatment on serum SOD activity in rats with $\mathrm{CCl}_{4}$-induced acute liver damage. ${ }^{*} P<$ 0.001 vs normal control; ${ }^{\#} P<0.001$ vs $\mathrm{CCl}_{4}$ control; mean (SD), $n=8$.
(Figures 1 and 2). This phenomenon was confirmed by histological changes (Figures $5 \mathrm{a}$ and $5 \mathrm{~b}$ ). Different from previous report (which showed that berberine has no curative effect on acute liver damage) [10], results from this study suggest that post-treatment with berberine may protect liver function. In addition, the histological sections of rat livers post-treated with berberine in Figure $5 \mathrm{c}$-e showed reduced incidence of liver lesions, hepatocyte swelling, leukocyte infiltrations and necrosis induced by $\mathrm{CCl}_{4}$ (Figures $5 \mathrm{a}$ and $5 \mathrm{~b}$ ). Histological evidence from this study supports the effectiveness of berberine to treat liver damage caused by $\mathrm{CCl}_{4}$.

Hwang et al. reported that berberine exhibited antioxidant property by its ability to quench free radicals of 1,1-diphenyl-1-picrylhydrazyl, decrease the leakage of lactate dehydrogenase and ALT and prevent the formation of malondialdehyde induced by t-BHP [11]. Janbaz and Gilani reported that post-treatment with berberine (4 $\mathrm{mg} / \mathrm{kg})$ after $\mathrm{CCl}_{4}$-induced hepatotoxicity exhibited no effect in reducing hepatic damage [10]. Sun et al., however, reported that berberine protected liver injury evidenced by decreased ALT and AST activities and that berberine's action was focused on liver fibrosis in $\mathrm{CCl}_{4}$-induced rats [12]. The apparent discrepancy between the two studies may be due to the dosages, animal species and animal models used. The present study found that berberine had both preventive and curative effects on $\mathrm{CCl}_{4}$-induced liver damage. Moreover, our findings suggest that dosages may be an important factor for curative effects of berberine. The dosage $(4 \mathrm{mg} / \mathrm{kg})$ used by Janbazour et al. was far below the effective dosage $(80-160 \mathrm{mg} / \mathrm{kg}$ ) reported in this study, which was determined according to our clinical experience [13] and was similar to the dosage reported by Sun et al. [12].

Pre-treatment of berberine significantly decreased both serum ALT and AST activities elevated by $\mathrm{CCl}_{4}$-induced hepatoxicity while serum SOD level significantly decreased (Figures 3 and 4). These results demonstrate the preventive hepatoprotective effects of berberine against liver damage induced by $\mathrm{CCl}_{4}$, further supported by the histological changes (Figure $5 \mathrm{f}$ ).

\section{Conclusion}

The present study finds that berberine possesses hepatoprotective activities against $\mathrm{CCl}_{4}$-induced hepatotoxicity in a dose-dependent manner. The heptoprotective activities are both preventive and curative. These findings were further supported by the histological changes in the liver. Berberine should be a lead for developing new drugs to treat drug/chemical-induced liver toxicity. 


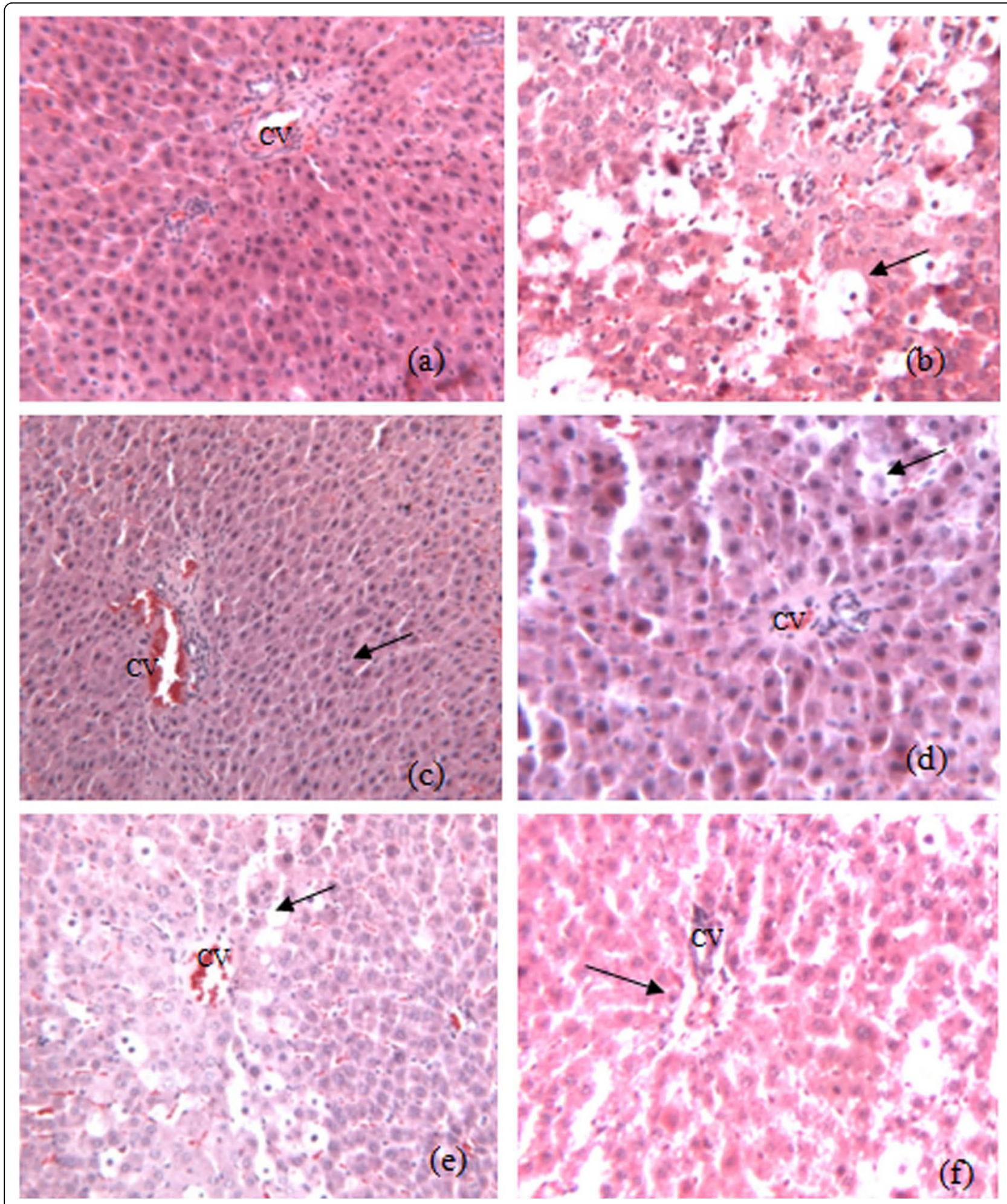

Figure 5 Photomicrography of liver sections of rats. a. liver sections of normal rats treated with olive oil vehicle only; $\mathbf{b}$. liver section of the control rat treated with $\mathrm{CCl}_{4}$ only; $\mathbf{c}$. liver section of the $\mathrm{CCl}_{4}$-treated rat post-treated by berberine at $160 \mathrm{mg} / \mathrm{kg}$ d. liver section of the $\mathrm{CCl}_{4}$ treated rat post-treated by berberine at $120 \mathrm{mg} / \mathrm{kg}$; e. liver section of the $\mathrm{CCl}_{4}$-treated rat post-treated by berberine at $80 \mathrm{mg} / \mathrm{kg} ; \mathbf{f}$. liver section of the $\mathrm{CCl}_{4}$-treated rat pre-treated by berberine at $120 \mathrm{mg} / \mathrm{kg}$ twice daily for two days (H\&E stain, original magnification $\times 100$ ). 
Table 1 Microscopic observation on protective and preventive effects of berberine against $\mathrm{CCl}_{4}$-induced acute liver damage in rats $(n=8)$

\begin{tabular}{|c|c|c|c|c|c|c|c|}
\hline Group & $\begin{array}{l}\text { Fatty } \\
\text { degeneration } \\
\text { Mean (SD) }\end{array}$ & $\begin{array}{l}\text { Vacoulisation } \\
\text { Mean (SD) }\end{array}$ & $\begin{array}{l}\text { Nuclei } \\
\text { Mean (SD) }\end{array}$ & $\begin{array}{l}\text { Hepatocyte } \\
\text { necrosis } \\
\text { Mean (SD) }\end{array}$ & $\begin{array}{l}\text { Inflammatory cells } \\
\text { infiltration } \\
\text { Mean (SD) }\end{array}$ & $\begin{array}{l}\text { Central vein and } \\
\text { portal triad } \\
\text { Mean (SD) }\end{array}$ & $\begin{array}{l}\text { Combined } \\
\text { score } \\
\text { Mean (SD) }\end{array}$ \\
\hline Normal & $0.6(0.3)$ & $0.3(0.2)$ & $1.3(0.3)$ & $0.5(0.1)$ & $0.7(0.3)$ & $2.2(0.6)$ & $1.2(0.3)$ \\
\hline $\mathrm{CCl}_{4}$ & $5.5(1.2)^{* *}$ & $4.8(0.4)^{* *}$ & $0.3(0.2) * *$ & $5.7(1.9)^{* *}$ & $5.5(1.5) * *$ & $0.4(0.2) * *$ & $4.7(0.9)^{\# \#}$ \\
\hline \multicolumn{8}{|c|}{$\begin{array}{l}\text { Post-treated with } \\
\text { berberine }\end{array}$} \\
\hline 80 mg/kg & $3.2(1.6)$ \#\# & $2.5(0.7){ }^{\# \#}$ & $1.7(0.3)$ \#\# & $2.2(0.4){ }^{\# \#}$ & $2.7(1.1) \#$ & $1.2(0.5)$ \#\# & $3.0(1.3) \# \#$ \\
\hline 120 mg/kg & $1.7(1.3)^{\# \#}$ & $1.8(0.2){ }^{\# \#}$ & $1.7(0.5){ }^{\# \#}$ & $1.6(0.8){ }^{\# \#}$ & $1.8(0.2){ }^{\# \#}$ & $2.1(0.6) \#$ & $1.8(0.9){ }^{\# \#}$ \\
\hline 160 mg/kg & $1.4(0.9){ }^{\# \#}$ & $1.2(0.4){ }^{\# \#}$ & $1.4(0.4) \#$ & $1.2(0.5){ }^{\# \#}$ & $1.1(0.4)^{\# \#}$ & $1.5(0.8){ }^{\# \#}$ & $1.1(0.8)$ \#\# \\
\hline \multicolumn{8}{|c|}{$\begin{array}{l}\text { Pre-treated with } \\
\text { berberine }\end{array}$} \\
\hline 120 mg/kg & $2.1(1.3) \#$ & $2.3(1.6) \#$ & $1.0(0.7)$ \#\# & $2.0(1.4)$ \#\# & $1.5(0.6) \# \#$ & $2.5(0.4)$ \#\# & $2.8(1.4)$ \#\# \\
\hline
\end{tabular}

${ }^{* *} P<0.001$ when compared with normal group; ${ }^{\#} P<0.001$ when compared with $\mathrm{CCl}_{4}$ group. The $P$ values higher than 0.001 were denoted after the means.

\section{Abbreviations}

ALT: alanine aminotransferase; AST: aspartate aminotransferase; $\mathrm{CCl}_{4}$ : Carbon tetrachloride; CRAE: Coptidis Rhizoma aqueous extract; H\&E: hematoxylin and eosin; ROS: reactive oxygen species; SOD: superoxide dismutase; $\mathrm{XO}$ : xanthine oxidase; CCD: Charge-coupled device

\section{Acknowledgements}

This study was financially supported by grants from the Research Council of the University of Hong Kong (200811159197, 200907176140), Pong Ding Yueng Endowment Fund for Education \& Research (20005274) and the Research Grants Committee (RGC) of Hong Kong (764708M). The authors are grateful to the support of Professors Yung-chi Cheng, Sun-Ping Lee, Chiming Che and Allan SY Lau. The authors would also like to express special thanks to Mr Keith Wong, Ms Cindy Lee and Mr Freddy Tsang for their technical support.

\section{Author details}

${ }^{1}$ School of Chinese Medicine, The University of Hong Kong, 10 Sassoon Road, Pokfulam, Hong Kong SAR, China. ²Department of Medicine, The University of Hong Kong, Queen Mary Hospital, Pokfulam Road, Hong Kong SAR, China. ${ }^{3}$ Department of Chemistry and Open Laboratory of Chemical Biology of the Institute of Molecular Technology for Drug Discovery and Synthesis, Faculty of Science, The University of Hong Kong, Pokfulam Road, Hong Kong SAR, China. ${ }^{4}$ Department of Medical Laboratory Science, Faculty of Health Sciences, Hokkaido University, Kita-12, Nishi-5, Kita-ku, Sapporo, Japan.

\section{Authors' contributions}

YF designed the study, conducted the experiments, analyzed the data and drafted the manuscript. KYS, XY and NW conducted the experiments, collected the data and helped draft the manuscript. MFY, CHL, YT and SK interpreted the data and revised the manuscript. All authors read and approved the final version of the manuscript.

\section{Competing interests}

The authors declare that they have no competing interests.

Received: 20 February 2010 Accepted: 18 September 2010 Published: 18 September 2010

\section{References}

1. Clawson GA: Mechanism of carbon tetrachloride hepatotoxicity. Pathol Immunopathol Res 1989, 8:104-112.

2. Recknagel RO, Glende EA, Dolak JA, Waller RL: Mechanism of carbon tetrachloride toxicity. Pharmacol Ther 1989, 43:139-154.

3. Tang J, Feng Y, Tsao S, Wang N, Curtain R, Wang Y: Berberine and Coptidis Rhizoma as novel antineoplastic agents: a review of traditional use and biomedical investigations. J Ethnopharmacol 2009, 126:5-17.
4. Ye X, Feng Y, Tong Y, Ng KM, Tsao SW, Lau GKK, Sze CW, Zhang Y, Tang J, Shen J, Kobayashi S: Hepatoprotective effects of Coptidis rhizoma aqueous extract on carbon tetrachloride-induced acute liver hepatotoxicity in rats. J Ethnopharmacol 2009, 124:130-136.

5. Yokozawa T, Ishida A, Cho EJ, Kim HY, Kashiwada Y, Ikeshiro Y: Coptidis Rhizoma: protective effects against peroxynitrite-induced oxidative damage and elucidation of its active components. J Pharm Pharmacol 2004, 56:547-556.

6. Kuo CL, Chi CW, Liu TY: The anti-inflammatory potential of berberine in vitro and in vivo. Cancer Lett 2004, 203:127-137.

7. Hsieh YS, Kuo WH, Lin TW, Chang HR, Lin TH, Chen PN, Chu SC: Protective effects of Berberine against Low-Density Lipoprotein (LDL) oxidation and oxidized LDL-Induced cytotoxicity on endothelial cells. J Agric Food Chem 2007, 55:10437-10445.

8. Zhu F, Qian C: Berberine chloride can ameliorate the spatial memory impairment and increase the expression of interleukin-1beta and inducible nitric oxide synthase in the rat model of Alzheimer's disease. BMC Neurosci 2006, 7:78.

9. Peng PL, Hsieh YS, Wang CJ, Hsu JL, Chou FP: Inhibitory effect of berberine on the invasion of human lung cancer cells via decreased productions of urokinase-plasminogen activator and matrix metalloproteinase-2. Toxicol Appl Pharmacol 2006, 214:8-15.

10. Janbaz KH, Gilani AH: Studies on preventive and curative effects of berberine on chemical-induced hepatotoxicity in rodents. Fitoterpia 2000, 71:25-33.

11. Hwang JM, Wang CJ, Chou FP, Tseng TH, Hsieh YS, Lin WL, Chu CY: Inhibitory effect of berberine on tert-butyl hydroperoxide-induced oxidative damage in rat liver. Arch Toxicol 2002, 76:664-670.

12. Sun X, Zhang X, Hu H, Lu Y, Chen J, Yasuda K, Wang H: Berberine inhibits hepatic stellate cell proliferation and prevents experimental liver fibrosis. Biol Pharm Bull 2009, 32:1533-1537.

13. Feng Y, Luo WQ, Zhu SQ: Explore new clinical application of Huanglian and corresponding compound prescriptions fromtheir traditional use. Zhongguo Zhong Yao Za Zhi 2008, 33:1221-1225.

doi:10.1186/1749-8546-5-33

Cite this article as: Feng et al:: Hepatoprotective effects of berberine on carbon tetrachloride-induced acute hepatotoxicity in rats. Chinese Medicine 2010 5:33. 